Algebra Univers. 70 (2013) 137-147

DOI $10.1007 / \mathrm{s} 00012-013-0245-0$

Published online July 25, 2013

(C) 2013 The Author(s)

Algebra Universalis

This article is published with open access at Springerlink.com

\title{
Menger algebras of $n$-place interior operations
}

\author{
Wieslaw A. Dudek and Valentin S. Trokhimenko
}

\begin{abstract}
Algebraic properties of $n$-place interior operations on a fixed set are described. Conditions under which a Menger algebra of rank $n$ can be represented by $n$-place interior operations are found.
\end{abstract}

\section{Introduction}

It is known [5] that on the topology on a set $A$ one can talk in the language of open sets, the language of closed sets, the language of interior operations (also called opening operations), or the language of closure operations. Various types of closure operations on algebraic systems and their applications are well described (see for example [3]). So a natural question is about a similar characterization of interior operations having applications in topology and economics. Such operations were first studied from an algebraic point of view by Vagner [7]. Kulik observed in [4] that the superposition of two interior operations is not always an interior operation and found conditions under which the composition of two interior operations of a given set $A$ is also an interior operation of this set. Moreover, he proved that a semigroup $S$ is isomorphic to a semigroup of interior operations of some set if and only if $S$ is idempotent and commutative.

Below, we introduce the concept of $n$-place interior operations and find conditions under which a Menger algebra of rank $n$ can be isomorphically represented by $n$-place interior operations of some set.

\section{Preliminaries}

Let $A$ be a nonempty set, $\mathfrak{P}(A)$ the family of all subsets of $A$, and $\mathcal{T}_{n}(\mathfrak{P}(A))$ the set of all $n$-place transformations of $\mathfrak{P}(A)$, i.e., maps $f: \prod^{n} \mathfrak{P}(A) \rightarrow$ $\mathfrak{P}(A)$, where $\prod^{n} \mathfrak{P}(A)$ denotes the $n$-th Cartesian power of the set $\mathfrak{P}(A)$. For arbitrary $f, g_{1}, \ldots, g_{n} \in \mathcal{T}_{n}(\mathfrak{P}(A))$, we define the $(n+1)$-ary composition $f\left[g_{1} \cdots g_{n}\right]$ by putting

$$
f\left[g_{1} \cdots g_{n}\right]\left(X_{1}, \ldots, X_{n}\right)=f\left(g_{1}\left(X_{1}, \ldots, X_{n}\right), \ldots, g_{n}\left(X_{1}, \ldots, X_{n}\right)\right)
$$

for all $X_{1}, \ldots, X_{n} \in \mathfrak{P}(A)$.

Presented by M. Jackson.

Received April 30, 2012; accepted in final form December 18, 2012.

2010 Mathematics Subject Classification: Primary: 20N15; Secondary: 08A62, 08A99.

Key words and phrases: Menger algebras, interior operations. 
The $(n+1)$-ary operation $\mathcal{O}:\left(f, g_{1}, \ldots, g_{n}\right) \mapsto f\left[g_{1} \cdots g_{n}\right]$ is called the Menger superposition of $n$-place functions (cf. [3, 6]). Then $\left(\mathcal{T}_{n}(\mathfrak{P}(A)), \mathcal{O}\right)$ is a Menger algebra in the sense of [2] and [3], i.e., the operation $\mathcal{O}$ satisfies the so-called superassociative law:

$$
f\left[g_{1} \cdots g_{n}\right]\left[h_{1} \cdots h_{n}\right]=f\left[g_{1}\left[h_{1} \cdots h_{n}\right] \cdots g_{n}\left[h_{1} \cdots h_{n}\right]\right],
$$

where $f, g_{i}, h_{i} \in \mathcal{T}_{n}(\mathfrak{P}(A))$, for $i=1, \ldots, n$.

We say that $n$-place transformation $f \in \mathcal{T}_{n}(\mathfrak{P}(A))$ is

- contractive if for any $X_{1}, \ldots, X_{n} \in \mathfrak{P}(A), f\left(X_{1}, \ldots, X_{n}\right) \subseteq X_{1} \cap \cdots \cap X_{n}$;

- idempotent if $f[f \cdots f]=f$;

- isotone if for any $X_{1}, \ldots, X_{n}, Y_{1}, \ldots, Y_{n} \in \mathfrak{P}(A)$,

$$
X_{1} \subseteq Y_{1} \wedge \cdots \wedge X_{n} \subseteq Y_{n} \Longrightarrow f\left(X_{1}, \ldots, X_{n}\right) \subseteq f\left(Y_{1}, \ldots, Y_{n}\right) ;
$$

- $\cup$-distributive if for all $X, Y, H_{1}, \ldots, H_{n} \in \mathfrak{P}(A)$ and $i=1, \ldots, n$,

$$
f\left(H_{1}^{i-1}, X \cup Y, H_{i+1}^{n}\right)=f\left(H_{1}^{i-1}, X, H_{i+1}^{n}\right) \cup f\left(H_{1}^{i-1}, Y, H_{i+1}^{n}\right),
$$

where $H_{s}^{r}$ means $H_{s}, \ldots, H_{r}$ for $s \leqslant r$.

- $\cap$-distributive if for all $X, Y, H_{1}, \ldots, H_{n} \in \mathfrak{P}(A)$ and $i=1, \ldots, n$,

$$
f\left(H_{1}^{i-1}, X \cap Y, H_{i+1}^{n}\right)=f\left(H_{1}^{i-1}, X, H_{i+1}^{n}\right) \cap f\left(H_{1}^{i-1}, Y, H_{i+1}^{n}\right) ;
$$

- full $\cup$-distributive if for any subsets $\left(X_{k}\right)_{k \in K}$ of $A$, all $H_{1}, \ldots, H_{n} \in \mathfrak{P}(A)$, and $i=1, \ldots, n$,

$$
f\left(H_{1}^{i-1}, \bigcup_{k \in K} X_{k}, H_{i+1}^{n}\right)=\bigcup_{k \in K} f\left(H_{1}^{i-1}, X_{k}, H_{i+1}^{n}\right) ;
$$

- full $\cap$-distributive if for any subsets $\left(X_{k}\right)_{k \in K}$ of $A$, all $H_{1}, \ldots, H_{n} \in \mathfrak{P}(A)$, and $i=1, \ldots, n$,

$$
f\left(H_{1}^{i-1}, \bigcap_{k \in K} X_{k}, H_{i+1}^{n}\right)=\bigcap_{k \in K} f\left(H_{1}^{i-1}, X_{k}, H_{i+1}^{n}\right) .
$$

It is not difficult to see that the contractivity of an $n$-place transformation $f \in \mathcal{T}_{n}(\mathfrak{P}(A))$ is equivalent to the system of conditions

$$
f\left(X_{1}, \ldots, X_{n}\right) \subseteq X_{i}, \text { for } i=1, \ldots, n .
$$

The isotonicity is equivalent to the system of $n$ implications

$$
X \subseteq Y \Longrightarrow f\left(H_{1}^{i-1}, X, H_{i+1}^{n}\right) \subseteq f\left(H_{1}^{i-1}, Y, H_{i+1}^{n}\right), \text { for } i=1, \ldots, n,
$$

where $X, Y, X_{1}, \ldots, X_{n}, H_{1}, \ldots, H_{n} \in \mathfrak{P}(A)$.

It is also easy to show that the Menger superposition of contractive (isotone) $n$-place transformations of $\mathfrak{P}(A)$ is again a contractive (isotone) $n$-place transformation of $\mathfrak{P}(A)$.

An $n$-place transformation of $\mathfrak{P}(A)$, which is contractive, idempotent, and isotone, is called an $n$-place interior operation or an $n$-place interior operator on the set $A$.

For $n=1$, this definition coincides with the definition of interior operations proposed by Vagner (see [7]). 


\section{Properties of $n$-place interior operations}

We start with the following characterization of $n$-place interior operations.

Theorem 3.1. For an $n$-place transformation $f$ of $\mathfrak{P}(A)$, the following conditions are equivalent:

(a) $f$ is an $n$-place interior operation on $A$;

(b) for all $X_{1}, \ldots, X_{n}, Y_{1}, \ldots, Y_{n} \in \mathfrak{P}(A)$, we have

$$
f\left(X_{1} \cap Y_{1}, \ldots, X_{n} \cap Y_{n}\right) \subseteq f\left(f\left(X_{1}^{n}\right), \ldots, f\left(X_{1}^{n}\right)\right) \cap f\left(Y_{1}^{n}\right) \cap Y_{1} \cap \cdots \cap Y_{n} .
$$

Proof. (a) $\Longrightarrow(\mathrm{b})$ : Suppose that $f$ is an $n$-place interior operation on $A$. Then by the contractivity of $f$, for $X_{i}, Y_{i} \in \mathfrak{P}(A)$, for $i=1, \ldots, n$, we have

$$
f\left(X_{1} \cap Y_{1}, \ldots, X_{n} \cap Y_{n}\right) \subseteq\left(X_{1} \cap Y_{1}\right) \cap \cdots \cap\left(X_{n} \cap Y_{n}\right) \subseteq Y_{1} \cap \cdots \cap Y_{n} .
$$

As $X_{i} \cap Y_{i} \subseteq Y_{i}, i=1, \ldots, n$, the isotonity of $f$ implies

$$
f\left(X_{1} \cap Y_{1}, \ldots, X_{n} \cap Y_{n}\right) \subseteq f\left(Y_{1}, \ldots, Y_{n}\right)=f\left(Y_{1}^{n}\right) .
$$

Similarly, $X_{i} \cap Y_{i} \subseteq X_{i}$ for $i=1, \ldots, n$ implies

$$
f\left(X_{1} \cap Y_{1}, \ldots, X_{n} \cap Y_{n}\right) \subseteq f\left(X_{1}, \ldots, X_{n}\right)=f\left(X_{1}^{n}\right) .
$$

Since $f\left(X_{1}^{n}\right)=f\left(f\left(X_{1}^{n}\right), \ldots, f\left(X_{1}^{n}\right)\right)$, from the above we obtain

$$
f\left(X_{1} \cap Y_{1}, \ldots, X_{n} \cap Y_{n}\right) \subseteq f\left(f\left(X_{1}^{n}\right), \ldots, f\left(X_{1}^{n}\right)\right),
$$

which together with (3.2) and (3.3) gives (3.1). Thus, (a) implies (b).

(b) $\Longrightarrow(\mathrm{a})$ : If an $n$-place transformation $f$ satisfies (3.1), then setting $X_{i}=Y_{i}$ for $i=1, \ldots, n$ in (3.1), we obtain

$$
f\left(X_{1}^{n}\right) \subseteq f\left(f\left(X_{1}^{n}\right), \ldots, f\left(X_{1}^{n}\right)\right) \cap f\left(X_{1}^{n}\right) \cap X_{1} \cap \cdots \cap X_{n} .
$$

So $f\left(X_{1}^{n}\right) \subseteq X_{1} \cap \cdots \cap X_{n}$, i.e., $f$ is contractive. In addition, (3.4) implies $f\left(X_{1}^{n}\right) \subseteq f\left(f\left(X_{1}^{n}\right), \ldots, f\left(X_{1}^{n}\right)\right)$. Since $f$ is contractive, we have

$$
f\left(f\left(X_{1}^{n}\right), \ldots, f\left(X_{1}^{n}\right)\right) \subseteq f\left(X_{1}^{n}\right) \cap \cdots \cap f\left(X_{1}^{n}\right)=f\left(X_{1}^{n}\right),
$$

which together with the previous inclusion proves that $f$ is idempotent.

If $X_{i} \subseteq Y_{i}$, then obviously $X_{i} \cap Y_{i}=X_{i}$. Hence, by (3.1), for $X_{i} \subseteq Y_{i}$ for $i=1, \ldots, n$, we have

$$
\begin{aligned}
f\left(X_{1}^{n}\right) & =f\left(X_{1} \cap Y_{1}, \ldots, X_{n} \cap Y_{n}\right) \\
& \subseteq\left(f\left(X_{1}^{n}\right), \ldots, f\left(X_{1}^{n}\right)\right) \cap f\left(Y_{1}^{n}\right) \cap Y_{1} \cap \cdots \cap Y_{n} \subseteq f\left(Y_{1}^{n}\right),
\end{aligned}
$$

which means that $f$ is isotone. Thus, $f$ is an $n$-place interior operation. So (b) implies (a).

Theorem 3.2. For an n-place transformation $f$ of $\mathfrak{P}(A)$, the following conditions are equivalent:

(i) $f$ is contractive and full $\cup$-distributive;

(ii) $f$ is contractive and $\cup$-distributive; 
(iii) for all $X_{1}, \ldots, X_{n} \in \mathfrak{P}(A)$, we have

$$
f\left(X_{1}^{n}\right)=f(A, \ldots, A) \cap X_{1} \cap \cdots \cap X_{n} .
$$

Proof. (i) $\Longrightarrow$ (ii): This is obvious.

(ii) $\Longrightarrow$ (iii): According to $\cup$-distributivity, for all subsets $X, H_{1}, \ldots, H_{n}$ of $A$ and $i=1, \ldots, n$ we have

$$
f\left(H_{1}^{i-1}, X, H_{i+1}^{n}\right) \cup f\left(H_{1}^{i-1}, X^{\prime}, H_{i+1}^{n}\right)=f\left(H_{1}^{i-1}, A, H_{i+1}^{n}\right),
$$

where $X^{\prime}=A \backslash X$. Then clearly

$$
\left(f\left(H_{1}^{i-1}, X, H_{i+1}^{n}\right) \cap X\right) \cup\left(f\left(H_{1}^{i-1}, X^{\prime}, H_{i+1}^{n}\right) \cap X\right)=f\left(H_{1}^{i-1}, A, H_{i+1}^{n}\right) \cap X .
$$

Since $f$ is contractive, we have $f\left(H_{1}^{i-1}, X, H_{i+1}^{n}\right) \subseteq X$, which implies that $f\left(H_{1}^{i-1}, X, H_{i+1}^{n}\right) \cap X=f\left(H_{1}^{i-1}, X, H_{i+1}^{n}\right)$. Similarly, $f\left(H_{1}^{i-1}, X^{\prime}, H_{i+1}^{n}\right) \subseteq X^{\prime}$ and $X^{\prime} \cap X=\varnothing$ imply $f\left(H_{1}^{i-1}, X^{\prime}, H_{i+1}^{n}\right) \cap X=\varnothing$. Thus, (3.6) has the form

$$
f\left(H_{1}^{i-1}, X, H_{i+1}^{n}\right)=f\left(H_{1}^{i-1}, A, H_{i+1}^{n}\right) \cap X .
$$

Using this identity, we obtain

$$
\begin{aligned}
& f\left(X_{1}, X_{2}, X_{3}, \ldots, X_{n}\right)=f\left(A, X_{2}, X_{3}, \ldots, X_{n}\right) \cap X_{1} \\
& =f\left(A, A, X_{3}, \ldots, X_{n}\right) \cap X_{2} \cap X_{1}=\ldots \\
& =f(A, A, A, \ldots, A) \cap X_{n} \cap \cdots \cap X_{2} \cap X_{1}=f(A, \ldots, A) \cap X_{1} \cap \cdots \cap X_{n} .
\end{aligned}
$$

So (ii) implies (iii).

(iii) $\Longrightarrow$ (i): That (3.5) implies $f\left(X_{1}, \ldots, X_{n}\right) \subseteq X_{1} \cap \cdots \cap X_{n}$ is not difficult to see. Thus, $f$ is contractive. Moreover, in this case we also have

$$
\begin{aligned}
& f(A, \ldots, A) \cap H_{1} \cap \cdots \cap H_{i-1} \cap\left(\bigcup_{k \in K} X_{k}\right) \cap H_{i+1} \cap \cdots \cap H_{n} \\
& =\bigcup_{k \in K} f\left(H_{1}^{i-1}, X_{k}, H_{i+1}^{n}\right)
\end{aligned}
$$

by (3.5). So $f$ is distributive with respect to the union. Thus (iii) implies (i), which completes the proof.

Corollary 3.3. Every $n$-place transformation $f$ on $\mathfrak{P}(A)$ satisfying $(3.5)$ is an $n$-place interior operation on $A$.

Proof. Any transformation satisfying (3.5) is clearly contractive. It is also idempotent, because

$$
\begin{aligned}
f\left(f\left(X_{1}^{n}\right), \ldots, f\left(X_{1}^{n}\right)\right) & =f(A, \ldots, A) \cap f\left(X_{1}^{n}\right) \\
& =f(A, \ldots, A) \cap f(A, \ldots, A) \cap X_{1} \cap \cdots \cap X_{n} \\
& =f(A, \ldots, A) \cap X_{1} \cap \cdots \cap X_{n}=f\left(X_{1}^{n}\right)
\end{aligned}
$$

for all $X_{1}, \ldots, X_{n} \in \mathfrak{P}(A)$.

For $X_{1} \subseteq Y_{1}, \ldots, X_{n} \subseteq Y_{n}$, we have $X_{1} \cap \cdots \cap X_{n} \subseteq Y_{1} \cap \cdots \cap Y_{n}$. Thus, $f(A, \ldots, A) \cap X_{1} \cap \cdots \cap X_{n} \subseteq f(A, \ldots, A) \cap Y_{1} \cap \cdots \cap Y_{n}$. So $f\left(X_{1}^{n}\right) \subseteq f\left(Y_{1}^{n}\right)$. Hence, $f$ is isotone. 
Corollary 3.4. Every (full) $\cup$-distributive n-place interior operation is (full) $\cap$-distributive.

Proof. Indeed, by Theorem 3.2, any $\cup$-distributive $n$-place interior operation $f$ on the set $A$ satisfies (3.5). Hence,

$$
\begin{aligned}
& f\left(H_{1}^{i-1}, X \cap Y, H_{i+1}^{n}\right) \\
& =f(A, \ldots, A) \cap H_{1} \cap \cdots \cap H_{i-1} \cap(X \cap Y) \cap H_{i+1} \cap \cdots \cap H_{n} \\
& =\left(f(A, \ldots, A) \cap H_{1} \cap \cdots \cap H_{i-1} \cap X \cap H_{i+1} \cap \cdots \cap H_{n}\right) \\
& \quad \cap\left(f(A, \ldots, A) \cap H_{1} \cap \cdots \cap H_{i-1} \cap Y \cap H_{i+1} \cap \cdots \cap H_{n}\right) \\
& =f\left(H_{1}^{i-1}, X, H_{i+1}^{n}\right) \cap f\left(H_{1}^{i-1}, Y, H_{i+1}^{n}\right)
\end{aligned}
$$

for $X, Y, H_{1}, \ldots, H_{n} \in \mathfrak{P}(A)$ and $i=1, \ldots, n$. Thus, $f$ is $\cap$-distributive.

Analogously, we can show that $f$ is full $\cap$-distributive.

\section{Compositions of $n$-place interior operations}

On the set $\mathcal{T}_{n}(\mathfrak{P}(A))$ of $n$-place transformations of the set $A$ we introduce the binary relation $\preceq$ defined by

$$
f \preceq g \Longleftrightarrow\left(\forall X_{1}, \ldots, X_{n}\right)\left(f\left(X_{1}^{n}\right) \subseteq g\left(X_{1}^{n}\right)\right) .
$$

It is easy to see that $\preceq$ is a partial order, i.e., it is reflexive, transitive, and antisymmetric.

Proposition 4.1. The relation $\preceq$ has the following properties:

(a) If $f \in \mathcal{T}_{n}(\mathfrak{P}(A))$ is contractive, then $f\left[g_{1} \cdots g_{n}\right] \preceq g_{i}$ for $i=1, \ldots, n$ and all $g_{1}, \ldots, g_{n} \in \mathcal{T}_{n}(\mathfrak{P}(A))$.

(b) If $f \in \mathcal{T}_{n}(\mathfrak{P}(A))$ is isotone, then

$$
g_{1} \preceq h_{1} \wedge \cdots \wedge g_{n} \preceq h_{n} \Longrightarrow f\left[g_{1} \cdots g_{n}\right] \preceq f\left[h_{1} \cdots h_{n}\right]
$$

for all $g_{1}, \ldots, g_{n}, h_{1}, \ldots, h_{n} \in \mathcal{T}_{n}(\mathfrak{P}(A))$.

(c) If $f \in \mathcal{T}_{n}(\mathfrak{P}(A))$ is isotone and $g \in \mathcal{T}_{n}(\mathfrak{P}(A))$ is contractive, then

$$
f[g \cdots g] \preceq f .
$$

Proof. (a): If $f \in \mathcal{T}_{n}(\mathfrak{P}(A))$ is contractive, then for all $X_{1}, \ldots, X_{n} \in \mathfrak{P}(A)$ and $i=1, \ldots, n$, we have $f\left[g_{1} \cdots g_{n}\right]\left(X_{1}^{n}\right)=f\left(g_{1}\left(X_{1}^{n}\right), \ldots, g_{n}\left(X_{1}^{n}\right)\right) \subseteq g_{i}\left(X_{1}^{n}\right)$. Hence, $f\left[g_{1} \cdots g_{n}\right] \preceq g_{i}$. So the first property is proved.

(b): If $f \in \mathcal{T}_{n}(\mathfrak{P}(A))$ is isotone and $g_{i} \preceq h_{i}$ for all $g_{i}, h_{i} \in \mathcal{T}_{n}(\mathfrak{P}(A))$ and all $i=1, \ldots, n$, then for all $X_{1}, \ldots, X_{n} \in \mathfrak{P}(A)$, we have $g_{i}\left(X_{1}^{n}\right) \subseteq h_{i}\left(X_{1}^{n}\right)$ for all $i=1, \ldots, n$; consequently, $f\left(g_{1}\left(X_{1}^{n}\right), \ldots, g_{n}\left(X_{1}^{n}\right)\right) \subseteq f\left(h_{1}\left(X_{1}^{n}\right), \ldots, h_{n}\left(X_{1}^{n}\right)\right)$. Therefore, $f\left[g_{1} \cdots g_{n}\right]\left(X_{1}^{n}\right) \subseteq f\left[h_{1} \cdots h_{n}\right]\left(X_{1}^{n}\right)$. So $f\left[g_{1} \cdots g_{n}\right] \preceq f\left[h_{1} \cdots h_{n}\right]$, which proves the second condition.

(c): This is a consequence of (a) and (b). 
Theorem 4.2. The Menger superposition of given n-place interior operations $f, g_{1}, \ldots, g_{n}$ defined on the set $A$ is an $n$-place interior operation on $A$ if and only if for each $i=1, \ldots, n$, we have

$$
g_{i}[f \cdots f]\left[g_{1} \cdots g_{n}\right]=f\left[g_{1} \cdots g_{n}\right] .
$$

Proof. $(\Longrightarrow)$ : Suppose that $f, g_{1}, \ldots, g_{n}$ and $f\left[g_{1} \cdots g_{n}\right]$ are $n$-place interior operations on $A$. Since each $g_{i}$ is contractive, according to (2.1) and Proposition 4.1, we obtain

$$
g_{i}[f \cdots f]\left[g_{1} \cdots g_{n}\right]=g_{i}\left[f\left[g_{1} \cdots g_{n}\right] \cdots f\left[g_{1} \cdots g_{n}\right]\right] \preceq f\left[g_{1} \cdots g_{n}\right] .
$$

On the other hand, using (2.1) and the fact that $f\left[g_{1} \cdots g_{n}\right]$ is an idempotent $n$-place transformation, we get

$$
\begin{aligned}
f\left[g_{1} \cdots g_{n}\right] & =f\left[g_{1} \cdots g_{n}\right]\left[f\left[g_{1} \cdots g_{n}\right] \cdots f\left[g_{1} \cdots g_{n}\right]\right] \\
& =f\left[g_{1}\left[f\left[g_{1} \cdots g_{n}\right] \cdots f\left[g_{1} \cdots g_{n}\right]\right] \cdots g_{n}\left[f\left[g_{1} \cdots g_{n}\right] \cdots f\left[g_{1} \cdots g_{n}\right]\right]\right] \\
& \preceq g_{i}\left[f\left[g_{1} \cdots g_{n}\right] \cdots f\left[g_{1} \cdots g_{n}\right]\right]=g_{i}[f \cdots f]\left[g_{1} \cdots g_{n}\right],
\end{aligned}
$$

which together with the previous inequality gives (4.1).

$(\Longleftarrow)$ : As mentioned above, Menger superposition preserves contractivity and isotonicity. We show that $f\left[g_{1} \cdots g_{n}\right]$ is idempotent. Indeed, according to (2.1) and (4.1), we have

$$
\begin{aligned}
& f\left[g_{1} \cdots g_{n}\right]\left[f\left[g_{1} \cdots g_{n}\right] \cdots f\left[g_{1} \cdots g_{n}\right]\right] \\
& =f\left[g_{1}\left[f\left[g_{1} \cdots g_{n}\right] \cdots f\left[g_{1} \cdots g_{n}\right]\right] \cdots g_{n}\left[f\left[g_{1} \cdots g_{n}\right] \cdots f\left[g_{1} \cdots g_{n}\right]\right]\right] \\
& =f\left[g_{1}[f \cdots f]\left[g_{1} \cdots g_{n}\right] \cdots g_{n}[f \cdots f]\left[g_{1} \cdots g_{n}\right]\right] \\
& =f\left[f\left[g_{1} \cdots g_{n}\right] \cdots f\left[g_{1} \cdots g_{n}\right]\right]=f[f \cdots f]\left[g_{1} \cdots g_{n}\right]=f\left[g_{1} \cdots g_{n}\right] .
\end{aligned}
$$

Thus, $f\left[g_{1} \cdots g_{n}\right]$ is an $n$-place interior operation.

\section{Algebras derived from their diagonal semigroups}

Recall (cf. [2], [3]) that a Menger algebra $(G, o)$ of rank $n$ is a nonempty set $G$ with an $(n+1)$-ary operation $o:\left(f, g_{1}, \ldots, g_{n}\right) \mapsto f\left[g_{1} \cdots g_{n}\right]$ satisfying the identity (2.1). On such algebra we can define a binary operation $*$ by setting $x * y=x[y \cdots y]$ for any $x, y \in G$. It is easy to see that $(G, *)$ is a semigroup. It is called the diagonal semigroup of $(G, o)$. In the case when

$$
f\left[g_{1} g_{2} \cdots g_{n}\right]=f * g_{1} * g_{2} * \cdots * g_{n},
$$

we say that a Menger algebra $(G, o)$ is derived from its diagonal semigroup $(G, *)$.

Proposition 5.1. For any n-place interior operations $f, g$ on the set $A$, the following three conditions are equivalent:

(a) $f * g$ is an $n$-place interior operation on $A$;

(b) $f * g=g * f * g$;

(c) $f * g=f * g * f$. 
Proof. (a) $\Longrightarrow$ (b): This implication follows from Theorem 4.2.

(b) $\Longrightarrow($ c): By Proposition 4.1, we have $f[g \cdots g] \preceq f$, i.e., $f * g \preceq f$. This together with (b) shows $f * g=g * f * g \preceq g * f$. So $f * g \preceq g * f$, which in view of Proposition 4.1 (b), gives $f * f * g \preceq f * g * f$. Hence, $f * g=f * f * g \preceq f * g * f \preceq f * g$, and consequently, $f * g=f * g * f$.

$(\mathrm{c}) \Longrightarrow(\mathrm{a})$ : Since Menger superposition preserves contractivity and isotonicity, then $f * g$ is an isotone and contractive $n$-place transformation. We show that it is idempotent. In fact,

$$
(f * g) *(f * g)=(f * g * f) * g=(f * g) * g=f *(g * g)=f * g .
$$

Thus, $f * g$ is an $n$-place interior operation.

\section{Characterizations of algebras of $n$-place interior operations}

An abstract characterization of Menger algebras of $n$-place interior operations is given in the following theorem.

Theorem 6.1. A Menger algebra $(G, o)$ of rank $n$ is isomorphic to a Menger algebra of n-place interior operations on some set if and only if it satisfies the following three identities

$$
\begin{aligned}
& x[x \cdots x]=x, \\
& x[y \cdots y]=y[x \cdots x], \\
& x\left[y_{1} \cdots y_{n}\right]=x\left[y_{1} \cdots y_{1}\right] \cdots\left[y_{n} \cdots y_{n}\right] .
\end{aligned}
$$

Proof. Necessity: Let $(\Phi, \mathcal{O})$, where $\Phi \subset \mathcal{T}_{n}(\mathfrak{P}(A))$, be a Menger algebra of $n$-place interior operations on the set $A$. Then obviously, $f[f \cdots f]=f$ for all $f \in \Phi$. Thus, the condition (6.1) is satisfied.

If $f, g \in \Phi$, then also $f[g \cdots g], g[f \cdots f] \in \Phi$. Therefore, $f * g$ and $g * f$ are $n$-place interior operations and by (b), (c) from Proposition 5.1, we have $f * g=g * f$. Thus, $f[g \cdots g]=g[f \cdots f]$. Hence, the condition (6.2) also is satisfied.

Further, using (2.1) and Theorem 4.2, for $f, g_{1}, \ldots, g_{n} \in \Phi$ and for each $i=1, \ldots, n$, we obtain

$g_{i} * f\left[g_{1} \cdots g_{n}\right]=g_{i}\left[f\left[g_{1} \cdots g_{n}\right] \cdots f\left[g_{1} \cdots g_{n}\right]\right]=g_{i}[f \cdots f]\left[g_{1} \cdots g_{n}\right]=f\left[g_{1} \cdots g_{n}\right]$.

Thus, $g_{i} * f\left[g_{1} \cdots g_{n}\right]=f\left[g_{1} \cdots g_{n}\right]$ for all $i=1, \ldots, n$. Consequently,

$$
\begin{aligned}
\left(f * g_{1}\right. & \left.* \cdots * g_{i}\right) * f\left[g_{1} \cdots g_{n}\right]=\left(f * g_{1} * \cdots * g_{i-1}\right) *\left(g_{i} * f\left[g_{1} \cdots g_{n}\right]\right) \\
& =\left(f * g_{1} * \cdots * g_{i-1}\right) * f\left[g_{1} \cdots g_{n}\right] \\
& \vdots \\
& =f * f\left[g_{1} \cdots g_{n}\right]=f\left[f\left[g_{1} \cdots g_{n}\right] \cdots f\left[g_{1} \cdots g_{n}\right]\right] \\
& =f[f \cdots f]\left[g_{1} \cdots g_{n}\right]=f\left[g_{1} \cdots g_{n}\right] .
\end{aligned}
$$


Hence,

$$
\left(f * g_{1} * \cdots * g_{i}\right) * f\left[g_{1} \cdots g_{n}\right]=f\left[g_{1} \cdots g_{n}\right]
$$

for every $i=1, \ldots, n$. Since $f\left[g_{1} \cdots g_{n}\right] \preceq g_{n}$ and

$$
\left(f * g_{1} * \cdots * g_{n-1}\right) * f\left[g_{1} \cdots g_{n}\right] \preceq\left(f * g_{1} * \cdots * g_{n-1}\right) * g_{n},
$$

by Proposition 4.1, the equality (6.4) means that

$$
f\left[g_{1} \cdots g_{n}\right] \preceq f * g_{1} * \cdots * g_{n} .
$$

By Proposition 4.1, we also have $f * g_{1} * \cdots * g_{n} \preceq f * g_{1} * \cdots * g_{i} \preceq g_{i}$ for all $i=1, \ldots, n$. Therefore, $f\left[\left(f * g_{1} * \cdots * g_{n}\right) \cdots\left(f * g_{1} * \cdots * g_{n}\right)\right] \preceq f\left[g_{1} \cdots g_{n}\right]$, i.e., $f *\left(f * g_{1} * \cdots * g_{n}\right) \preceq f\left[g_{1} \cdots g_{n}\right]$, whence by $f * f=f$, we obtain

$$
f * g_{1} * \cdots * g_{n} \preceq f\left[g_{1} \cdots g_{n}\right] .
$$

This, together with (6.5), gives $f\left[g_{1} \cdots g_{n}\right]=f * g_{1} * \cdots * g_{n}$. Thus, the condition (6.3) is satisfied too.

Sufficiency: Let $(G, o)$ be a Menger algebra of rank $n$ satisfying all the conditions of the theorem and let $(G, *)$ be its diagonal semigroup. Consider a binary relation $\omega$ defined on the set $G$ as follows: $\omega=\{(x, y) \mid x * y=y\}$. Since the diagonal semigroup $(G, *)$ is semilattice, the relation $\omega$ is reflexive, transitive, and antisymmetric. So the relation $\omega$ is just the usual ordering on a semilattice (treated as a join semilattice), and $\omega\langle x\rangle=\{y \in G \mid(x, y) \in \omega\}$ is just the upset in this ordering.

We show that

$$
\omega\left\langle x\left[y_{1} \cdots y_{n}\right]\right\rangle=\omega\langle x\rangle \cap \omega\left\langle y_{1}\right\rangle \cap \cdots \cap \omega\left\langle y_{n}\right\rangle
$$

for $x, y_{1}, \ldots, y_{n} \in G$.

Since the diagonal semigroup $(G, *)$ is semilattice, the equation $\omega\langle x * y\rangle=$ $\omega\langle x\rangle \cap \omega\langle y\rangle$ holds in $(G, *)$.

Using this equation and (6.3) we obtain

$$
\begin{aligned}
\omega\left\langle x\left[y_{1} \cdots y_{n}\right]\right\rangle & =\omega\left\langle x * y_{1} * \cdots * y_{n}\right\rangle=\omega\left\langle x *\left(y_{1} * \cdots * y_{n}\right)\right\rangle \\
& =\omega\langle x\rangle \cap \omega\left\langle y_{1} *\left(y_{2} * \cdots * y_{n}\right)\right\rangle=\omega\langle x\rangle \cap \omega\left\langle y_{1}\right\rangle \cap \omega\left\langle y_{2} * \cdots * y_{n}\right\rangle \\
& =\cdots=\omega\langle x\rangle \cap \omega\left\langle y_{1}\right\rangle \cap \cdots \cap \omega\left\langle y_{n}\right\rangle,
\end{aligned}
$$

which proves $(6.6)$.

Consider the set $\Phi=\left\{f_{g} \mid g \in G\right\} \subseteq \mathcal{T}_{n}(\mathfrak{P}(G))$ of all $n$-place operations $f_{g}$ defined by

$$
f_{g}\left(X_{1}^{n}\right)=\omega\langle g\rangle \cap X_{1} \cap \cdots \cap X_{n}
$$

and the map $P: g \mapsto f_{g}$. 
Clearly, $f_{g}(G, \ldots, G)=\omega\langle g\rangle$. Thus, $f_{g}\left(X_{1}^{n}\right)=f_{g}(G, \ldots, G) \cap X_{1} \cap \cdots \cap X_{n}$. Hence, by Corollary $3.3, f_{g}$ is an $n$-place interior operation on the set $G$. Moreover, for all $g, g_{1}, \ldots, g_{n}$ and $X_{1}, \ldots, X_{n} \in \mathfrak{P}(G)$, we have

$$
\begin{aligned}
& f_{g}\left[f_{g_{1}} \cdots f_{g_{n}}\right]\left(X_{1}^{n}\right)=f_{g}\left(f_{g_{1}}\left(X_{1}^{n}\right), \ldots, f_{g_{n}}\left(X_{1}^{n}\right)\right) \\
& =\omega\langle g\rangle \cap f_{g_{1}}\left(X_{1}^{n}\right) \cap \cdots \cap f_{g_{n}}\left(X_{1}^{n}\right) \\
& =\omega\langle g\rangle \cap\left(\omega\left\langle g_{1}\right\rangle \cap X_{1} \cap \cdots \cap X_{n}\right) \cap \cdots \cap\left(\omega\left\langle g_{n}\right\rangle \cap X_{1} \cap \cdots \cap X_{n}\right) \\
& =\left(\omega\langle g\rangle \cap \omega\left\langle g_{1}\right\rangle \cap \cdots \cap \omega\left\langle g_{n}\right\rangle\right) \cap X_{1} \cap \cdots \cap X_{n} \\
& \stackrel{(6.6)}{=} \omega\left\langle g\left[g_{1} \cdots g_{n}\right]\right\rangle \cap X_{1} \cap \cdots \cap X_{n}=f_{g\left[g_{1} \cdots g_{n}\right]}\left(X_{1}^{n}\right) .
\end{aligned}
$$

Thus, $P\left(g\left[g_{1} \cdots g_{n}\right]\right)=P(g)\left[P\left(g_{1}\right) \cdots P\left(g_{n}\right)\right]$, i.e., $P$ is a homomorphism of $G$ onto $\Phi$.

Suppose now that $P\left(g_{1}\right)=P\left(g_{2}\right)$, where $g_{1}, g_{2} \in G$. Then $f_{g_{1}}=f_{g_{2}}$, i.e., $f_{g_{1}}\left(X_{1}^{n}\right)=f_{g_{2}}\left(X_{1}^{n}\right)$ for all $X_{1}, \ldots, X_{n} \in \mathfrak{P}(G)$. Hence, $\omega\left\langle g_{1}\right\rangle \cap X_{1} \cap \cdots \cap X_{n}=$ $\omega\left\langle g_{2}\right\rangle \cap X_{1} \cap \cdots \cap X_{n}$, which for $X_{1}=\cdots=X_{n}=G$, gives $\omega\left\langle g_{1}\right\rangle=\omega\left\langle g_{2}\right\rangle$. Since $\omega$ is an antisymmetric relation, from the above we conclude $g_{1}=g_{2}$. Thus, $P$ is a bijection of $G$ onto $\Phi$. This means that a Menger algebra $(G, o)$ of rank $n$ is isomorphic to the constructed Menger algebra $(\Phi, \mathcal{O})$ of $n$-place interior operations.

Corollary 6.2. A Menger algebra $(G, o)$ of rank $n$ is isomorphic to a Menger algebra of n-place interior operations on some set if and only if it is derived from an idempotent commutative semigroup.

The above corollary says that a Menger algebra isomorphic to a Menger algebra of $n$-place interior operations is derived from an idempotent commutative semigroup. Since a diagonal semigroup of a group-like Menger algebra is a group (see [1] or [3]), a group-like Menger algebra isomorphic to a Menger algebra of $n$-place interior operations has only one element. In view of Corollary 6.2 , it is obvious that two Menger algebras of $n$-place interior operations are isomorphic if and only if their diagonal semigroups are isomorphic.

According to Theorem 3.2 and Corollary 3.4, each $n$-place interior operation defined by $(6.7)$ is (full) $\cup$-distributive and (full) $\cap$-distributive. Therefore, we have the following corollary.

Corollary 6.3. For a Menger algebra $(G, o)$ of rank $n$ the following conditions are equivalent:

- $(G, o)$ is derived from an idempotent commutative semigroup.

- $(G, o)$ is isomorphic to a Menger algebra of contractive (full) $\cup$-distributive $n$-place transformations on some set.

- $(G, o)$ is isomorphic to a Menger algebra of (full) $\cup$-distributive $n$-place interior operations on some set.

- $(G, o)$ is isomorphic to a Menger algebra of (full) $\cup$-distributive and $\cap$ distributive n-place interior operations on some set. 


\section{Semigroups of interior operations}

Menger algebras of rank $n=1$ are (binary) semigroups. So, as a consequence of our results, we obtain some useful facts for semigroups.

Recall that Vagner (cf. [7]) defined interior operations on the set $A$ as contractive, idempotent, and isotone transformations of $\mathfrak{P}(A)$. This definition coincides with our definition for $n=1$. So, as a consequence of Theorem 3.1, we obtain the following corollary.

Corollary 7.1. A transformation $f$ of $\mathfrak{P}(A)$ is an interior operation on the set $A$ if and only if

$$
f(X \cap Y) \subseteq f(f(X)) \cap f(Y) \cap Y
$$

is valid for all $X, Y \in \mathfrak{P}(A)$.

From Theorem 3.2 and Corollary 3.3, we obtain

Corollary 7.2. For a transformation $f$ of $\mathfrak{P}(A)$, the following conditions are equivalent:

- $f$ is contractive and full $\cup$-distributive;

- $f$ is contractive and $\cup$-distributive;

- for every $X \in \mathfrak{P}(A)$, we have

$$
f(X)=f(A) \cap X .
$$

Corollary 7.3. Any transformation $f$ of $\mathfrak{P}(A)$ satisfying $(7.1)$ is an interior operation on $A$.

As a consequence of Corollary 3.4, we obtain

Corollary 7.4. Every (full) $\cup$-distributive interior operation is (full) $\cap$-distributive.

Putting $n=1$ in Theorem 4.2, we obtain one of the main results of the paper [4]:

Corollary 7.5. The composition $f \circ g$ of two interior operations defined on the same set is an interior operation on this set if and only if $f \circ g=f \circ g \circ f$.

The other main result of [4] is a consequence of our Corollary 6.3, which for $n=1$ has the following form:

Corollary 7.6. For a semigroup $(G, \cdot)$, the following statements are equivalent:

- $(G, \cdot)$ is an idempotent commutative semigroup;

- $(G, \cdot)$ is isomorphic to a semigroup of contractive and (full) $\cup$-distributive transformations on some set; 
- $(G, \cdot)$ is isomorphic to a semigroup of $($ full $) \cup$-distributive interior operations on some set.

- $(G, \cdot)$ is isomorphic to a semigroup of $($ full $) \cup$-distributive and $\cap$-distributive interior operations on some set.

From this, we obtain the following:

Corollary 7.7 (Podluzhnyak and Kulik [4]). A semigroup $(G, \cdot)$ is isomorphic to some semigroup of interior operations on some set $A$ if and only if it is idempotent and commutative.

\section{REFERENCES}

[1] Dudek, W.A.: On group-like Menger $n$-groupoids, Radovi Matematički (Sarajevo), 2, 81-98 (1986)

[2] Dudek, W.A., Trokhimenko, V.S.: Menger algebras of multiplace functions, Centrul Ed. USM, Chişinău (2006) (Russian)

[3] Dudek, W.A., Trokhimenko, V.S.: Algebras of multiplace functions, Walter de Gruyter GmbH \& Co. KG, Berlin/Boston (2012)

[4] Kulik, V.T., Podluzhnyak, H.G.: Abstract characterization of a semigroup of interior operations, Naukovi Zapiski, ser. Fiz. Mat. (Vinnitsa) 1, 341-346 (2002) (Ukrainian)

[5] Kuratowski, K.: Topology, vol. 1, new edition, Warszawa (1966)

[6] Schein, B.M., Trohimenko, V.S.: Algebras of multiplace functions, Semigroup Forum 17, 1-64 (1979)

[7] Vagner, V.V.: Representation of ordered semigroups, Mat. Sbornik 38(80), 203-240 (1956) (Russian)

\section{Wieslaw A. Dudek}

Institute of Mathematics and Computer Science, Wroclaw University of Technology, 50-370 Wroclaw, Poland

e-mail: wieslaw.dudek@pwr.wroc.pl

\section{VAlentin S. Trokhimenko}

Department of Mathematics, Pedagogical University, 21100 Vinnitsa, Ukraine e-mail: vtrokhim@gmail.com

Open Access This article is distributed under the terms of the Creative Commons Attribution License which permits any use, distribution, and reproduction in any medium, provided the original author(s) and the source are credited. 